\title{
Asymmetric Ionic Conditions Generate Large Membrane Curvatures
}

\author{
Marzieh Karimi, ${ }^{\dagger}$ Jan Steinkühler, ${ }^{\dagger}$ Debjit Roy, ${ }^{\dagger, \S}$ Raktim Dasgupta, ${ }^{\dagger,}$ Reinhard Lipowsky, ${ }^{\dagger}$ \\ and Rumiana Dimova* ${ }^{*}+$ (1) \\ ${ }^{\dagger}$ Department of Theory and Bio-Systems, Max Planck Institute of Colloids and Interfaces, Science Park Golm, 14424 Potsdam, \\ Germany \\ ${ }^{\ddagger}$ Laser Biomedical Applications Section, Raja Ramanna Centre for Advanced Technology, 452013 Indore, India
}

\section{Supporting Information}

ABSTRACT: Biological membranes possess intrinsic asymmetry. This asymmetry is associated not only with leaflet composition in terms of membrane species but also with differences in the cytosolic and periplasmic solutions containing macromolecules and ions. There has been a long quest for understanding the effect of ions on the physical and morphological properties of membranes. Here, we elucidate the changes in the mechanical properties of membranes exposed to asymmetric buffer conditions and the associated curvature generation. As a model system, we used giant unilamellar vesicles (GUVs) with asymmetric salt and sugar solutions on the two sides of the membrane. We aspirated

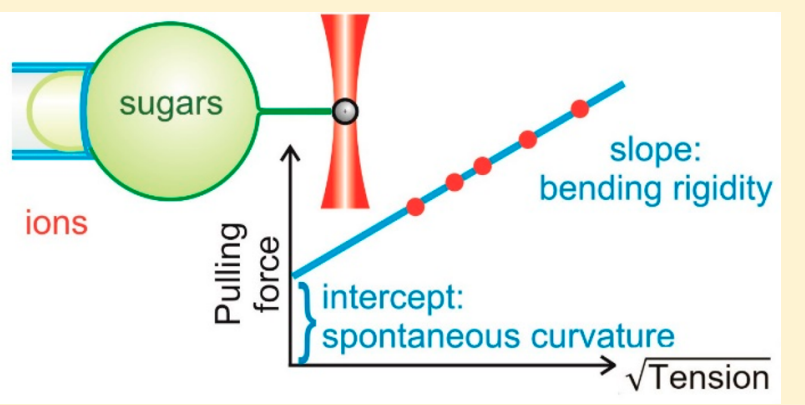
the GUVs into micropipettes and attached small beads to their membranes. An optical tweezer was used to exert a local force on a bead, thereby pulling out a membrane tube from the vesicle. The assay allowed us to measure the spontaneous curvature and the bending rigidity of the bilayer in the presence of different ions and sugar. At low sugar/salt (inside/out) concentrations, the membrane spontaneous curvature generated by $\mathrm{NaCl}$ and $\mathrm{KCl}$ is close to zero, but negative in the presence of $\mathrm{LiCl}$. In the latter case, the membrane bulges away from the salt solution. At high sugar/salt conditions, the membranes were observed to become more flexible and the spontaneous curvature was enhanced to even more negative values, comparable to those generated by some proteins. Our findings reveal the reshaping role of alkali chlorides on biomembranes.

KEYWORDS: Spontaneous curvature, asymmetric membranes, bending rigidity, membrane nanotubes, protein-free curvature generation, giant vesicle

Biological membranes are seldom flat but instead often exhibit strongly curved morphology. The shapes of cells and cellular organelles are highly conserved, suggesting that shape and membrane morphology are crucial for life. Deformations from the molecularly preferred curvature cost significant energy, often exceeding $10 k_{\mathrm{B}} T$, and yet membrane shape transformations are ubiquitous during cellular and organelle functions. The preferred or spontaneous curvature of membranes is determined by the asymmetry across and within the bilayer, ${ }^{1,2}$ and asymmetric conditions across the membrane are a hallmark of cellular life. However, the exact values of the spontaneous biomembrane curvature, and how cells control this important parameter, are poorly understood. Membrane shape changes are often sustained by the action of proteins, ${ }^{3}$ but this pathway is costly in terms of energy and molecular material. Ions, however, are abundant in the intra- and extraluminal space of cells and organelles and it is not clear whether and how they affect the membrane morphology and whether cells employ them as a cheap way of controlling membrane shape.

Mammalian cells maintain ion concentration differences by a system of ion channels that enable the cell to regulate the flow of ions across the membrane. Changing sodium and potassium ion concentrations allow nerve impulses to be transmitted down a nerve cell. Ions are responsible for the maintenance of the cellular homeostasis, proper $\mathrm{pH}$, osmotic pressure and water distribution in different fluid compartments of the body. ${ }^{4}$ They are also essential for regulating the proper function of the heart and other muscles. These are some examples of important functions of sodium and potassium that make them crucial elements in cells. In addition to sodium and potassium, lithium ions $\left(\mathrm{Li}^{+}\right)$play an important role in metabolism, neural communication, and cell proliferation. ${ }^{5,6}$ However, cellular processes controlled by lithium are not thoroughly studied, although low levels of lithium have shown beneficial effect on living organisms; e.g., lithium is a powerful drug in the treatment of manic depression. ${ }^{7,8}$

In conditions where the membrane is exposed to asymmetric concentration of different particles on both sides, the membrane prefers to bulge toward one of the aqueous compartments. This response results from molecular interactions, i.e., either adsorption or depletion. These interactions

Received: September 4, 2018

Revised: November 19, 2018

Published: November 20, 2018 
may have different effects on the bilayer structure. An adsorbed ion or solute, for example, may insert into the leaflet and push the lipids apart, thereby increasing the effective molecular area. Alternatively, an adsorbed species could also lead to local condensation of lipids, thereby reducing the effective molecular area. This behavior is described by the spontaneous (preferred) curvature of the membrane. It can be positive or negative if the membrane bends away from or toward the lumen, respectively. ${ }^{1,9}$ There has been a long quest to understand the effect of ions on the physical and morphological properties of the membrane. Considering the strong ion-lipid interactions supported by abundant evidence for adsorption of ions to the membrane (see, e.g., ref 10 and references therein) and, in particular, the recent findings on the impact of ion trans-membrane asymmetry on the membrane phase state, ${ }^{11}$ we set to investigate the effect of ion asymmetry on the mechanical properties of the membranes. We used giant unilamellar vesicles (GUVs) as a model membrane system. ${ }^{12-14}$ Their large sizes (in the range of tens of microns) enable us to manipulate the vesicle membranes and visualize the membrane response using optical microscopy. We aimed at investigating and quantifying the effect of $\mathrm{Na}^{+}, \mathrm{K}^{+}$, and $\mathrm{Li}^{+}$and their transmembrane asymmetry on the mechanical properties of phosphatidylcholine (POPC) model membranes.

Experimental Section. Vesicle Preparation. GUVs were prepared using the electroformation technique from $2 \mathrm{mM}$ of POPC and 0.1-0.2 mol \% of 1,2-dioleoyl-sn-glycero-3phosphoethanolamine- $N$-(cap biotinyl). Additionally, $0.1 \mathrm{~mol}$ $\%$ Texas Red 1,2-dihexadecanoyl-sn-glycero-3-phosphoethanolamine (Texas-Red-DHPE) was added for fluorescence imaging of the vesicles, see section S1 in the Supporting Information.

Experimental Setup. The tube-pulling setup is a combination of the micromanipulation and optical tweezers. ${ }^{15,16}$ Micromanipulation was performed using micropipettes of $\sim 7$ $\mu \mathrm{m}$ inner diameters for the aspiration and setting the membrane tension of GUVs (section S2 in the Supporting Information). The optical tweezers setup ${ }^{16}$ was calibrated by application of known hydrodynamic forces on the beads and subsequent imaging of the bead position in the trap; see section S3 in the Supporting Information). This calibration was later used to estimate the forces when the bead is displaced from the trap center by the pulled nanotube. Curve fitting and data analysis were preformed using image analysis implemented in MATLAB $2018 \mathrm{~b}^{15}$ and Origin 2018.

Results and Discussion. We prepared GUVs composed of POPC doped with fluorescent lipid by electroformation as described in the Experimental Section and section S1 in the Supporting Information. The vesicles were grown in sugar solutions, which were in this way encapsulated in the GUVs. Subsequent 1:20 dilution of the GUVs in slightly hypotonic salt solutions allowed the study of sugar-salt solution asymmetry on the lipid membrane (the inverse condition of salt inside and sugar outside was not feasible as no suitable vesicles could be produced for the explored salt concentrations). The GUV morphologies in asymmetric solution conditions were imaged with confocal microscopy. In solutions of rather low salt concentrations up to approximately $30 \mathrm{mM}$ of $\mathrm{NaCl}$, the GUVs exhibited mostly smooth, nearly spherical, membranes (Figure 1a, Table 1, condition a). Such vesicle morphologies indicate negligible membrane spontaneous curvature and are typical for GUVs in nearly symmetric solution conditions (data not shown). Strikingly, $\mathrm{LiCl}$ solution of similar concentration results in GUVs with highly curved
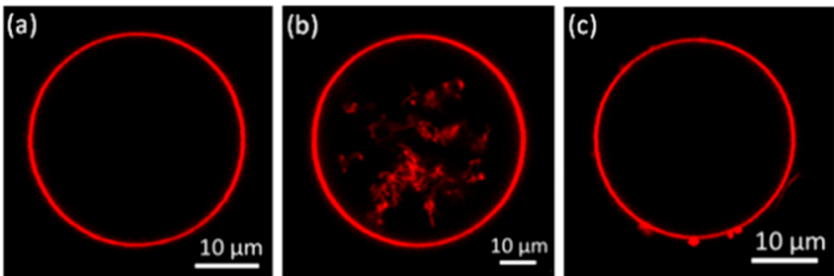

Figure 1. Confocal cross sections of different POPC GUVs with their two leaflets exposed to different solutions of sucrose and salts. GUVs were fluorescently labeled with $0.1 \mathrm{~mol} \%$ Texas-Red-DHPE and classified as (a) exhibiting no structures, (b) containing numerous nanotubes inside the vesicle, or (c) exhibiting structures (small buds, tubes, folds, or even adhering vesicles) pointing to the outside. Depending on the concentration difference of the salts on the two sides of the membrane, the relative frequency of each morphology changed. The results are summarized in Table 1.

Table 1. Statistics of Internal and External Structures (Compare Figure 1) for GUVs Exposed to Different Solutions of Sucrose and Salt

\begin{tabular}{llccc}
\multicolumn{2}{c}{$\begin{array}{l}\text { asymmetry } \\
\text { conditions }\end{array}$} & no structures & internal structures & external structures \\
a & low $\mathrm{NaCl}$ & $82 \%$ & $6 \%$ & $2 \%$ \\
$b$ & high $\mathrm{NaCl}$ & $29 \%$ & $30 \%$ & $41 \%$ \\
c & low $\mathrm{KCl}$ & $34 \%$ & $39 \%$ & $27 \%$ \\
d & high $\mathrm{KCl}$ & $6 \%$ & $77 \%$ & $17 \%$ \\
e & low $\mathrm{LiCl}$ & $20 \%$ & $69 \%$ & $10 \%$
\end{tabular}

${ }^{a}$ GUVs were grown in $54 \mathrm{mM}$ sucrose (inside) and diluted to 28.5 $\mathrm{mM} \mathrm{NaCl}$ and $2.7 \mathrm{mM}$ sucrose (outside); the total number of vesicles studied for this asymmetry was $n=50 .{ }^{b}$ GUVs with $260 \mathrm{mM}$ sucrose inside and $142.5 \mathrm{mM} \mathrm{NaCl}$ plus $13 \mathrm{mM}$ sucrose outside; $n=130$. ${ }^{c}$ GUVs with $114 \mathrm{mM}$ sucrose inside and $57 \mathrm{mM} \mathrm{KCl}$ plus $5.7 \mathrm{mM}$ sucrose outside; $n=58$. ${ }^{d}$ GUVs with $260 \mathrm{mM}$ sucrose inside and $142.5 \mathrm{mM} \mathrm{KCl}$ plus $13 \mathrm{mM}$ sucrose outside; $n=135$. ${ }^{e} \mathrm{GUVs}$ with 54 $\mathrm{mM}$ sucrose inside and $28.5 \mathrm{mM} \mathrm{LiCl}$ plus $2.7 \mathrm{mM}$ sucrose outside; $n$ $=85$. For all measurements, the given fractions have an uncertainty of about $15 \%$. The observed external structures include small buds and tubes, but also adhering vesicles.

membrane segments (nanotubes) pointing to the vesicle interior with diameters below the optical resolution (Figure $1 b$, Table 1, condition e). Direct observations of individual vesicles upon solution exchange showed the formation of the tubes induced by $\mathrm{LiCl}$ asymmetry; see Figure $\mathrm{S} 1$ in the Supporting Information. They were reminiscent of cylindrical and necklace-like tubes observed in studies with membranes exposed to polymer asymmetry. ${ }^{17,18}$ Increasing $\mathrm{NaCl}$ and $\mathrm{KCl}$ concentrations up to physiological levels $(150 \mathrm{mM})$ leads to a similar tendency for inward pointing nanotubes (Table 1 , conditions $\mathrm{b}$ and $\mathrm{d}$ ); note that the fraction of external structures also increases, which results from not only outward membrane protrusions but also adhesion of smaller vesicles. In principle, optical observation of tubes or buds allows for direct quantification of the membrane spontaneous curvature. ${ }^{2,18,19}$ However, adhesion of small vesicles to the GUVs (Figure 1c, which would be counted as external structures in Table 1), most prominent at high $\mathrm{NaCl}$ concentrations, interferes with clear assessment of the spontaneous curvature from such morphological studies alone. High $\mathrm{LiCl}$ concentrations were not investigated due to the strong adhesion of GUVs to each other.

To quantify the spontaneous curvature, we applied the more quantitative method of pulling of membrane nanotubes from 
the GUV membrane (see, e.g., refs 15 and 20). In this method, GUVs are aspirated with a micropipette, which controls the membrane tension $\Sigma_{\text {asp }}$ of the vesicles by changing the hydrostatic pressure difference applied by changing the height of a water reservoir connected to the pipet, see section S2 in the Supporting Information. A $2 \mu \mathrm{m}$ streptavidin bead trapped by optical tweezers was stuck to the vesicle and used to pull a membrane nanotube with $10 \mu \mathrm{m}$ length from the GUV. In this configuration, the pulling force can be estimated from the position of the bead in the optical trap which was measured by optical microscopy; see section S3 in the Supporting Information. The pulling force $f$ for an outward tube is given by $^{1,15}$

$$
f=2 \pi \sqrt{2 \kappa \Sigma_{\text {asp }}}-4 \pi \kappa m
$$

where $\kappa$ is the bending rigidity and $m$ is the membrane spontaneous curvature. Thus, by measuring the force for different membrane tension set by the pipet, one can deduce the bending rigidity form the slope of the data (first term in eq 1 ) and the spontaneous curvature from the intercept (second term). Figure 2 represents the experiment schematic and images of vesicles with a pulled membrane nanotube.

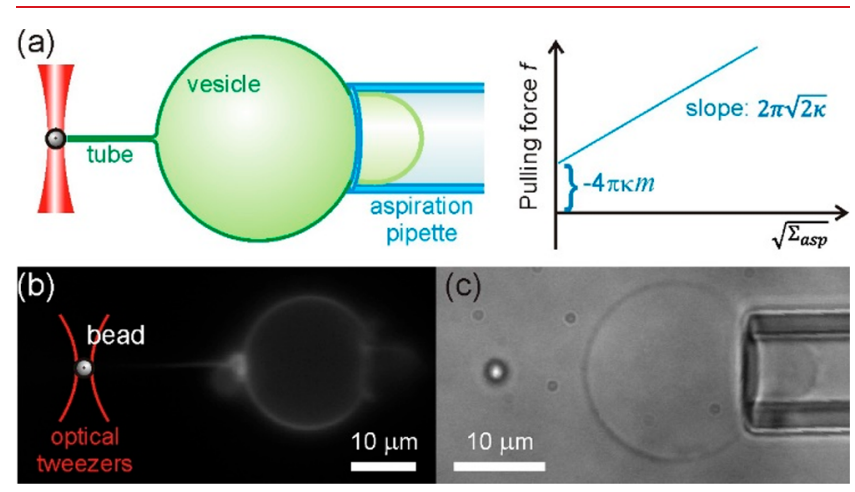

Figure 2. Experimental assay for pulling out a nanotube from a GUV: (a) sketch of the setup and principle of measurement and (b) epifluorescence and (c) bright-field images of an aspirated vesicle with a pulled tube.

The streptavidin-coated beads were injected into the experimental chamber locally via a syringe and were moved into the proximity of a GUV using the optical tweezers (see Experimental Section and section S2). A freely fluctuating GUV was aspirated into the glass capillary, a bead stuck to it, and a nanotube was pulled out by translating the vesicle away. Tube pulling experiments started with low concentration of $\mathrm{NaCl}$ outside the membrane, which was identified as the minimal salt concentration where bead-membrane adhesion was still efficient. As determined in the morphological studies of GUVs, this concentration was used further as a reference to compare our data to conditions that induce higher membrane asymmetry. Note that controls with symmetric sugar conditions were not possible as beads were not adhering to the membrane and thus tubes could not be pulled in the absence of salt. In all experimental conditions, vesicles were studied at suction pressures between $\sim 20$ and $\sim 49 \mathrm{~Pa}$. The membrane tension of the vesicles at each pressure could be assessed from the geometry of the aspirated vesicle and the applied pressure difference; see Supporting Information section S2. By careful calibration of the optical trap, the force exerted on the bead holding the nanotubes was determined from the optically measured bead displacement (see Experimental Section and Supporting Information sections S3 and S4). When tube pulling forces were measured, the trapped bead was displaced to the edge of the trap, i.e., away from the linear region of the trapping force field. This allowed us, for the same trap beam power, to measure higher forces than when the linear trapping force region was used. This is an advantageous approach as in this way the heating of the bead and the attached membrane is reduced. ${ }^{21,22}$ Moreover, considering possible variations of sizes of the trapped latex beads, we calibrated the trap stiffness for each individual bead before using it for pulling tubes. An approach combining the equipartition method and viscous drag method was used (see Supporting Information section S4).

By fitting all force vs tension data points to a linear fit following eq 1 , the mean bending rigidity of vesicles with low $\mathrm{NaCl}$ asymmetry (Figure 3a) was estimated as $34.79 \pm 0.81$ $k_{\mathrm{B}} T$. This value is well within the range of data reported for POPC membranes ${ }^{23}$ and in particular for POPC vesicles in the presence of $\mathrm{NaCl}$ as measured from fluctuation analysis. ${ }^{24,25}$ The spontaneous curvature of the membrane determined from the $y$-axis intercept of the fitted line was found to be $0.66 \pm$ $0.30 \mu \mathrm{m}^{-1}$; note that fitting individual measurements and subsequent averaging yields $0.09 \pm 0.68 \mu \mathrm{m}^{-1}$; see Table S1. This spontaneous curvature is negligible (comparable to the mean curvature of the GUVs), which is consistent with the spherical nontubulated morphologies observed in the microscopy images statistics (Table 1 ).

Identical experiments were performed on vesicles exposed to high asymmetry of physiological concentration $(150 \mathrm{mM})$ of $\mathrm{NaCl}$ outside and isotonic sucrose inside the vesicles. Six vesicles were studied in this condition and the mean bending rigidity was estimated to be $23.60 \pm 0.60 k_{\mathrm{B}} T$ (Figure $3 \mathrm{a}$ ). Comparing the bending rigidity in the two explored $\mathrm{NaCl}$ conditions, we conclude that the bending rigidity decreases by increasing $\mathrm{NaCl}$ (and sugar) concentration, confirming a trend reported in refs 24 and 25 (note that salts are reported to rigidify bilayer stacks (see, e.g., ref 26), which underlies the difference in the behavior of single membranes as in giant vesicles and multiple layers as in multilayered systems, each exploring lipid-to-ion ratios that differ by orders of magnitude). As a reminder, at high salt asymmetry, the sugar concentration inside the vesicles is also increased and sugars have been also shown to decrease the membrane bending rigidity (see overview in ref 23) presumably because of membrane thinning in this concentration range. ${ }^{27}$ It can be also seen from Figure $3 a$ that while the intercept of the linear fit under low asymmetry is zero within the fitting accuracy (blue shaded region), the intercept at high $\mathrm{NaCl}$ asymmetry implies nonzero spontaneous curvature. We estimated this negative spontaneous curvature as $-8.74 \pm 0.50 \mu \mathrm{m}^{-1}$, again consistent with the spontaneous appearance of membrane tubes with diameters below optical resolution $(<0.5 \mu \mathrm{m})$ observed in the morphology studies (Table 1). The values obtained for the spontaneous curvature imply that it would stabilize cylindrical tubes with diameters around $110 \mathrm{~nm}$. Measuring the tube diameter using fluorescence intensity as done in refs 28 and 29 was not feasible, as our microscope setup was equipped for epifluorescence only where bleaching poses a problem.

In some of the experiments at high $\mathrm{NaCl}$ asymmetry, the measured spontaneous curvature was found close to zero, but upon inspection of the vesicle population with phase contrast microscopy and employing an encapsulated water-soluble 

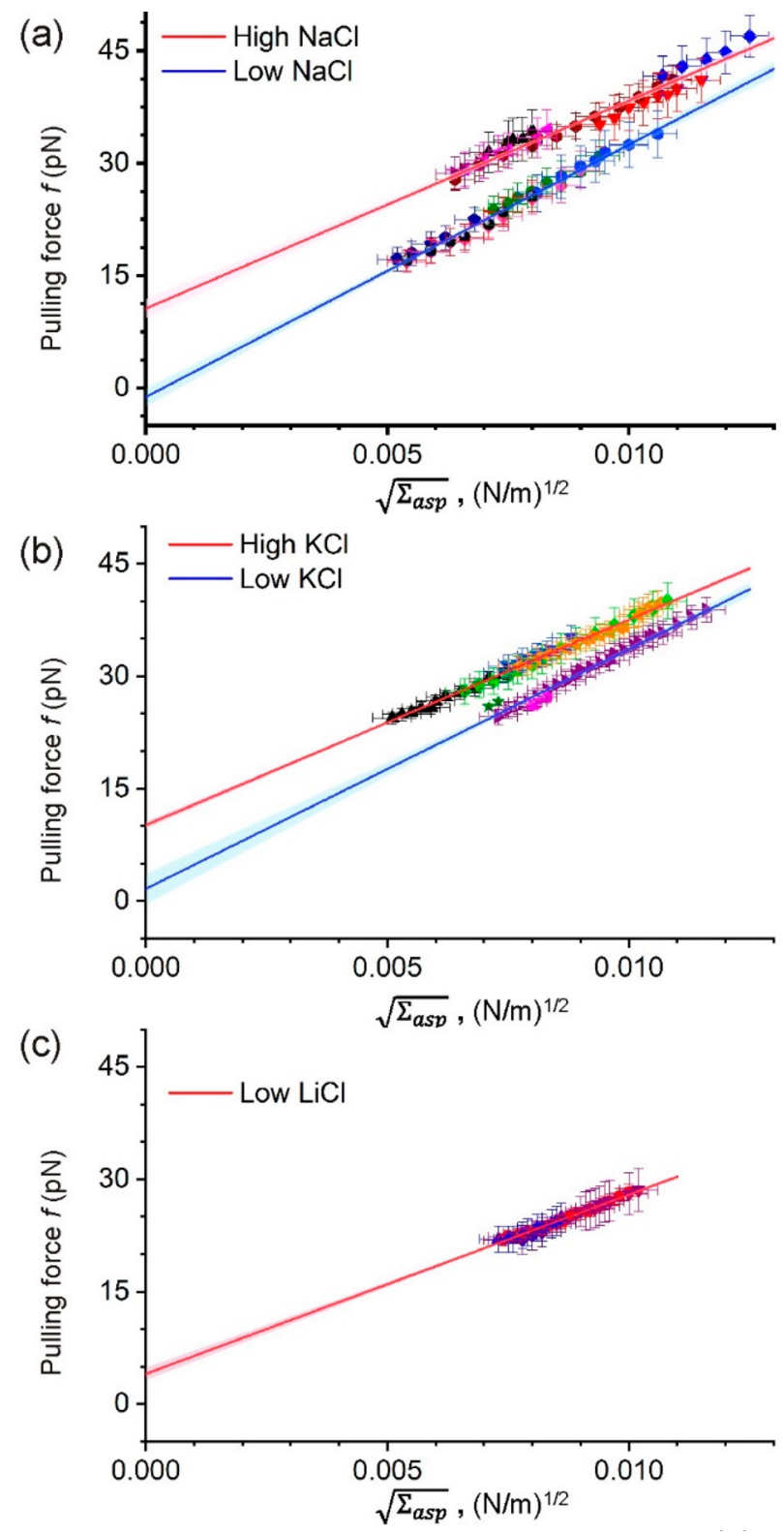

Figure 3. Results for out tubes showing the pulling force $(f)$ vs the square root of the membrane tension $\Sigma_{\text {asp }}$ for vesicles exposed to low asymmetry (blue linear fits) and high asymmetry (red linear fits) of sucrose inside and (a) $\mathrm{NaCl}$ outside, (b) $\mathrm{KCl}$ outside, and (c) $\mathrm{LiCl}$ outside the vesicles (the respective solution concentrations are given in the caption of Table 1). Differently colored symbols reflect measurements on different vesicles from different preparations. The solid lines are fits following eq 1 , yielding the material properties of the membrane, i.e., the bending rigidity and spontaneous curvature as schematically illustrated in Figure 2a.

fluorophore, we found that this is because vesicles have leaked and the asymmetry has been compromised; see Supporting Information section S5.

Similar experiments were performed for asymmetric $\mathrm{KCl}$ solutions. Concentration-wise, the low-asymmetry condition here does not correspond to that of low $\mathrm{NaCl}$ asymmetry as higher concentrations of $\mathrm{KCl}$ were required to ensure the beads sticking to the membrane. The respective bending rigidity values were $31.87 \pm 1.08 k_{\mathrm{B}} T$ and $23.11 \pm 0.24 k_{\mathrm{B}} T$ for low and high asymmetries, indicating softening of the membrane by $\mathrm{KCl}$ as observed for $\mathrm{NaCl}$. Similarly, increasing

$\mathrm{KCl}$ asymmetry was found to induce significant negative spontaneous curvature on the membranes; see Figure $3 \mathrm{~b}$ and data summarized in Table 2 . To restate, the bending rigidity

Table 2. Mean Values and Standard Deviations of the Bending Rigidity and the Spontaneous Curvature of Vesicle Membranes Exposed to Asymmetric Solutions of Sucrose and Salt As Described in the Caption of Table $1^{a}$

\begin{tabular}{lcc} 
condition & $\kappa\left(k_{\mathrm{B}} T\right)$ & $m\left(\mu \mathrm{m}^{-1}\right)$ \\
\hline low $\mathrm{NaCl}$ & $34.79 \pm 0.81$ & $0.66 \pm 0.30$ \\
high $\mathrm{NaCl}$ & $23.60 \pm 0.60$ & $-8.74 \pm 0.50$ \\
low $\mathrm{KCl}$ & $31.87 \pm 1.08$ & $-1.00 \pm 0.59$ \\
high $\mathrm{KCl}$ & $23.11 \pm 0.24$ & $-8.47 \pm 0.24$ \\
low $\mathrm{LiCl}$ & $17.52 \pm 0.34$ & $-4.45 \pm 0.45$
\end{tabular}

${ }^{a}$ Each value is obtained from a fit to all data for a given buffer composition. For values obtained from individual fits and subsequent averaging, see section S6 and Tables S1-S5 in the Supporting Information. The exact salt and sugar concentration conditions are given in Table 1.

and spontaneous curvature values are comparable for vesicles exposed to two different salts, i.e., $\mathrm{NaCl}$ and $\mathrm{KCl}$ in two different concentration ranges; i.e., each of these alkali chlorides affects the membrane similarly in terms of softening and curvature generation.

Finally, we also explored the effect of $\mathrm{LiCl}$ asymmetry. Even though physiological concentrations of lithium are in the (sub)millimolar range, studies on axonal remodeling and growth cone spreading (see, e.g., ref 30) explore concentrations on the order of $10 \mathrm{mM}$. To compare our results on $\mathrm{NaCl}$ asymmetry, we explored $\mathrm{LiCl}$ asymmetry at the same ion concentration; see Figure 3C and Table 1 for exact conditions. Even at this low $\mathrm{LiCl}$ concentration the bending rigidity is considerably decreased and a significant negative spontaneous curvature was measured consistent with microscopy observations (Table 1). All experiments are summarized in Table 2.

To confirm the effect of the salts on the lipid bilayers, the membrane bending rigidity was also measured via fluctuation analysis; ${ }^{31}$ see section S7 in the Supporting Information. The values of bending rigidity were estimated to be $29.8 \pm 3.7 k_{\mathrm{B}} T$ and $19.2 \pm 5.0 k_{\mathrm{B}} T$ for vesicles exposed to low asymmetry of $\mathrm{KCl}$ and $\mathrm{LiCl}$, respectively. These results are in good agreement with the bending rigidity values obtained from tube pulling experiments in Table 2 .

Conclusions. In this paper, we used a combination of optical tweezers and micropipette manipulation of giant vesicles to pull out lipid nanotubes and measure fundamental mechanical properties of the membranes. We focused on the effect of ionic asymmetry across the membrane as present in cells. The vesicles were exposed to different concentrations of sucrose (inside) and three different types of salts (outside), namely, $\mathrm{NaCl}, \mathrm{KCl}$, and $\mathrm{LiCl}$. Measurements of the tube pulling force and membrane tension of the vesicles, allowed us to assess the bending rigidity and spontaneous curvatures generated in the above conditions. The magnitude for the spontaneous curvature generated at high $\mathrm{NaCl}$ and $\mathrm{KCl}$ asymmetry is lower than values measured on membranes exposed to BAR domain proteins ${ }^{32}\left(m^{-1} \sim 20-100 \mathrm{~nm}\right)$ and comparable to that of membranes with asymmetric distribution of gangliosides, ${ }^{15,33}$ polymers, ${ }^{18}$ and more importantly, divalent ions ${ }^{34,35}\left(m^{-1} \sim 100-500 \mathrm{~nm}\right)$. The sign of the spontaneous curvature generated by calcium reported in refs 34 and 35 is 
controversial; one group reported that binding of calcium ions to these membranes generates positive spontaneous curvature, ${ }^{34}$ whereas measurements of another group at the same conditions yielded negative spontaneous curvature. ${ }^{35}$ Our results for $\mathrm{NaCl}$ and $\mathrm{KCl}$ corroborate negative spontaneous curvature at high salt concentration. Indeed, similarly to direct calcium-induced inward tubulation shown in ref 36 , we do observe more abundant internal tubes in these vesicles (Table 1 ). Thus, in the presence of salt solutions, vesicle membranes tend to curve away from the exterior salt solution attaining a negative spontaneous curvature. Presumably, the ions that adsorb to the membrane lead to local condensation of lipids, thereby reducing the effective molecular area of the leaflet exposed to them.

Interestingly, increasing salt asymmetries correlated with the change in the values of both the bending rigidity and spontaneous curvature. Generally, sugars are thought to soften membranes via thinning of the bilayers ${ }^{27}$ while alkali ions, except for lithium, are considered as not interacting with the membrane. ${ }^{37}$ As suggested by simulations and NMR data, ${ }^{37}$ we thus speculate that the spontaneous curvature is generated by sodium and potassium ion depletion from the bilayer (outer leaflet) while the adsorbed lithium (similarly to the effect of calcium) leads to local condensation of lipids, thereby reducing the effective molecular area of the leaflet exposed to them. From fluorescence lifetime measurements ${ }^{38}$ we attempted to distinguish the condensing effect of lithium ions from the depletion of sodium and potassium ions from the membrane; see section S8 in the Supporting Information. The lifetime of the used dye was altered only in the presence of $\mathrm{LiCl}$ (corresponding to the low $\mathrm{LiCl}$ asymmetry condition explored here) but not in the presence of $\mathrm{NaCl}$ and $\mathrm{KCl}$ solutions (in both high and low asymmetry conditions). We thus conclude that our speculations for the depletion of sodium and potassium ions from the membrane as well as for the condensing effect of lithium are reasonable. Presumably, molecular dynamics simulation studies will be able to further characterize the mechanism of curvature generation by monovalent salt asymmetry. In our experiments, the effect of the ions may also be enhanced by thinning of the monolayer facing the sugar solution (sugars are believed to laterally expand the leaflet ${ }^{27}$ ), all together driving the generation of the negative spontaneous curvature. One significant result to be emphasized here is that the bending rigidity and negative curvature generated by both $\mathrm{NaCl}$ and $\mathrm{KCl}$ are comparable. While the vesicle membrane has almost zero spontaneous curvature at low concentration of these salts, low concentration of $\mathrm{LiCl}$ induces a negative curvature that is approximately equivalent to half the value measured for the high asymmetry conditions with $\mathrm{NaCl}$ and $\mathrm{KCl}$. In a number of studies, lithium has been shown to have an inhibitory role on proteins (see, e.g., refs 8, 30, and 39 and references therein), leading to the conclusion that neuronal remodeling is predominantly governed by the activity of these proteins. Here, we show that biomembrane reshaping might result not solely from protein inhibition by lithium but also by the direct action of $\mathrm{LiCl}$ on membrane rigidity and spontaneous curvature resulting from the applied asymmetric solutions.

\section{ASSOCIATED CONTENT}

\section{S Supporting Information}

The Supporting Information is available free of charge on the ACS Publications website at DOI: 10.1021/acs.nanolett. 8 b03584.

Discussion of vesicle preparation and observation, micropipette manipulation, and optical trapping and calibration (including figures of epifluorescence images of one GUV; apparatus setup; trapped particle image, trajectory, and displacement; trap stiffness vs camera exposure time and particle number; and bead off-center displacement); tables of bending rigidity and spontaneous curvature data from individual vesicles; fluctuation analysis; fluorescence lifetime measurements (PDF)

\section{AUTHOR INFORMATION}

\section{Corresponding Author}

*Rumiana Dimova. E-mail: dimova@mpikg.mpg.de.

ORCID $\odot$

Reinhard Lipowsky: 0000-0001-8417-8567

Rumiana Dimova: 0000-0002-3872-8502

\section{Present Address}

${ }^{\S}$ Department of Cell Biology and Physiology, Washington University School of Medicine, St. Louis, MO 63110, USA.

\section{Author Contributions}

R.D., J.S., and M.K. designed the experiments. R.L. proposed the project. M.K. and J.S. performed the experiments. R. Dimova, D.R., J.S., M.K., and R. Dasgupta developed the trap calibration assay. All authors wrote the manuscript.

\section{Notes}

The authors declare no competing financial interest.

\section{ACKNOWLEDGMENTS}

This work is part of the MaxSynBio consortium which was jointly funded by the Federal Ministry of Education and Research of Germany and the Max Planck Society.

\section{REFERENCES}

(1) Lipowsky, R. Spontaneous tubulation of membranes and vesicles reveals membrane tension generated by spontaneous curvature. Faraday Discuss. 2013, 161, 305-331.

(2) Bassereau, P.; Jin, R.; Baumgart, T.; Deserno, M.; Dimova, R.; Frolov, V. A.; Bashkirov, P. V.; Grubmüller, H.; Jahn, R.; Risselada, H. J.; Johannes, L.; Kozlov, M. M.; Lipowsky, R.; Pucadyil, T. J.; Zeno, W. F.; Stachowiak, J. C.; Stamou, D.; Breuer, A.; Lauritsen, L.; Simon, C.; Sykes, C.; Voth, G. A.; Weikl, T. R. The 2018 biomembrane curvature and remodeling roadmap. J. Phys. D: Appl. Phys. 2018, 51 (34), 343001

(3) Zimmerberg, J.; Kozlov, M. M. How proteins produce cellular membrane curvature. Nat. Rev. Mol. Cell Biol. 2006, 7, 9.

(4) Pohl, H. R.; Wheeler, J. S.; Murray, H. E. Sodium and Potassium in Health and Disease. In Interrelations between Essential Metal Ions and Human Diseases; Sigel, A., Sigel, H., Sigel, R. K. O., Eds.; Springer: Dordrecht The Netherlands, 2013; pp 29-47.

(5) Shahzad, B.; Mughal, M. N.; Tanveer, M.; Gupta, D.; Abbas, G. Is lithium biologically an important or toxic element to living organisms? An overview. Environ. Sci. Pollut. Res. 2017, 24 (1), 103115.

(6) Williams, R.; Ryves, W. J.; Dalton, E. C.; Eickholt, B.; Shaltiel, G.; Agam, G.; Harwood, A. J. A molecular cell biology of lithium. Biochem. Soc. Trans. 2004, 32, 799-802.

(7) Pasternak, C. A. Monovalent Cations in Biological Systems; CRC Press, 1990. 
(8) Klein, P. S.; Melton, D. A. A molecular mechanism for the effect of lithium on development. Proc. Natl. Acad. Sci. U. S. A. 1996, 93 (16), 8455.

(9) Lipowsky, R. Coupling of bending and stretching deformations in vesicle membranes. Adv. Colloid Interface Sci. 2014, 208, 14-24.

(10) Klasczyk, B.; Knecht, V.; Lipowsky, R.; Dimova, R. Interactions of Alkali Metal Chlorides with Phosphatidylcholine Vesicles. Langmuir 2010, 26 (24), 18951-18958.

(11) Kubsch, B.; Robinson, T.; Lipowsky, R.; Dimova, R. Solution Asymmetry and Salt Expand Fluid-Fluid Coexistence Regions of Charged Membranes. Biophys. J. 2016, 110 (12), 2581-2584.

(12) Dimova, R.; Aranda, S.; Bezlyepkina, N.; Nikolov, V.; Riske, K. A.; Lipowsky, R. A practical guide to giant vesicles. Probing the membrane nanoregime via optical microscopy. J. Phys.: Condens. Matter 2006, 18 (28), S1151-S1176.

(13) Walde, P.; Cosentino, K.; Engel, H.; Stano, P. Giant Vesicles: Preparations and Applications. ChemBioChem 2010, 11 (7), 848-865.

(14) Dimova, R. Giant Vesicles: A Biomimetic Tool for Membrane Characterization. In Advances in Planar Lipid Bilayers and Liposomes, Iglič, A., Ed.; Academic Press, 2012; Vol. 16, pp 1-50.

(15) Dasgupta, R.; Miettinen, M. S.; Fricke, N.; Lipowsky, R.; Dimova, R. The glycolipid GM1 reshapes asymmetric biomembranes and giant vesicles by curvature generation. Proc. Natl. Acad. Sci. U. S. A. 2018, 115, 5756-5761.

(16) Kraikivski, P.; Pouligny, B.; Dimova, R. Implementing both short- and long-working-distance optical trappings into a commercial microscope. Rev. Sci. Instrum. 2006, 77 (11), 113703.

(17) Li, Y.; Lipowsky, R.; Dimova, R. Membrane nanotubes induced by aqueous phase separation and stabilized by spontaneous curvature. Proc. Natl. Acad. Sci. U. S. A. 2011, 108 (12), 4731-4736.

(18) Liu, Y.; Agudo-Canalejo, J.; Grafmüller, A.; Dimova, R.; Lipowsky, R. Patterns of Flexible Nanotubes Formed by LiquidOrdered and Liquid-Disordered Membranes. ACS Nano 2016, 10 (1), 463-474.

(19) Nikolov, V.; Lipowsky, R.; Dimova, R. Behavior of giant vesicles with anchored DNA molecules. Biophys. J. 2007, 92 (12), $4356-4368$.

(20) Sorre, B.; Callan-Jones, A.; Manneville, J. B.; Nassoy, P.; Joanny, J. F.; Prost, J.; Goud, B.; Bassereau, P. Curvature-driven lipid sorting needs proximity to a demixing point and is aided by proteins. Proc. Natl. Acad. Sci. U. S. A. 2009, 106 (14), 5622-5626.

(21) Liu, Y.; Cheng, D. K.; Sonek, G. J.; Berns, M. W.; Chapman, C. F.; Tromberg, B. J. Evidence for localized cell heating induced by infrared optical tweezers. Biophys. J. 1995, 68 (5), 2137-2144.

(22) Bolognesi, G.; Friddin, M. S.; Salehi-Reyhani, A.; Barlow, N. E.; Brooks, N. J.; Ces, O.; Elani, Y. Sculpting and fusing biomimetic vesicle networks using optical tweezers. Nat. Commun. 2018, 9 (1), 1882.

(23) Dimova, R. Recent developments in the field of bending rigidity measurements on membranes. Adv. Colloid Interface Sci. 2014, 208 (0), 225-234.

(24) Bouvrais, H. Mechanical properties of giant vesicle membranes investigated by flickering technique. Ph.D. thesis, University of Southern Denmark, Odense, 2011.

(25) Bouvrais, H. Bending Rigidities of Lipid Bilayers: Their Determination and Main Inputs in Biophysical Studies. In Advances in Planar Lipid Bilayers and Liposomes; Iglič, A., Ed.; Academic Press, 2012; Vol. 15, pp 1-75.

(26) Pabst, G.; Hodzic, A.; Strancar, J.; Danner, S.; Rappolt, M.; Laggner, P. Rigidification of neutral lipid bilayers in the presence of salts. Biophys. J. 2007, 93 (8), 2688-2696.

(27) Andersen, H. D.; Wang, C. H.; Arleth, L.; Peters, G. H.; Westh, $\mathrm{P}$. Reconciliation of opposing views on membrane-sugar interactions. Proc. Natl. Acad. Sci. U. S. A. 2011, 108 (5), 1874-1878.

(28) Sorre, B.; Callan-Jones, A.; Manzi, J.; Goud, B.; Prost, J.; Bassereau, P.; Roux, A. Nature of curvature coupling of amphiphysin with membranes depends on its bound density. Proc. Natl. Acad. Sci. U. S. A. 2012, 109 (1), 173-178.
(29) Aimon, S.; Callan-Jones, A.; Berthaud, A.; Pinot, M.; Toombes, G. E. S.; Bassereau, P. Membrane Shape Modulates Transmembrane Protein Distribution. Dev. Cell 2014, 28 (2), 212-218.

(30) Hall, A. C.; Lucas, F. R.; Salinas, P. C. Axonal Remodeling and Synaptic Differentiation in the Cerebellum Is Regulated by WNT-7a Signaling. Cell 2000, 100 (5), 525-535.

(31) Gracià, R. S.; Bezlyepkina, N.; Knorr, R. L.; Lipowsky, R.; Dimova, R. Effect of cholesterol on the rigidity of saturated and unsaturated membranes: fluctuation and electrodeformation analysis of giant vesicles. Soft Matter 2010, 6 (7), 1472-1482.

(32) McMahon, H. T.; Gallop, J. L. Membrane curvature and mechanisms of dynamic cell membrane remodelling. Nature 2005, 438 (7068), 590-596.

(33) Bhatia, T.; Agudo-Canalejo, J.; Dimova, R.; Lipowsky, R. Membrane Nanotubes Increase the Robustness of Giant Vesicles. ACS Nano 2018, 12 (5), 4478-4485.

(34) Simunovic, M.; Lee, K. Y. C.; Bassereau, P. Celebrating Soft Matter's 10th anniversary: screening of the calcium-induced spontaneous curvature of lipid membranes. Soft Matter 2015, 11 (25), 5030-5036.

(35) Graber, Z. T.; Shi, Z.; Baumgart, T. Cations induce shape remodeling of negatively charged phospholipid membranes. Phys. Chem. Chem. Phys. 2017, 19 (23), 15285-15295.

(36) Ali Doosti, B.; Pezeshkian, W.; Bruhn, D. S.; Ipsen, J. H.; Khandelia, H.; Jeffries, G. D. M.; Lobovkina, T. Membrane Tubulation in Lipid Vesicles Triggered by the Local Application of Calcium Ions. Langmuir 2017, 33 (41), 11010-11017.

(37) Catte, A.; Girych, M.; Javanainen, M.; Loison, C.; Melcr, J.; Miettinen, M. S.; Monticelli, L.; Määttä, J.; Oganesyan, V. S.; Ollila, O. H. S.; Tynkkynen, J.; Vilov, S. Molecular electrometer and binding of cations to phospholipid bilayers. Phys. Chem. Chem. Phys. 2016, 18 (47), 32560-32569.

(38) Packard, B. S.; Wolf, D. E. Fluorescence lifetimes of carbocyanine lipid analogs in phospholipid bilayers. Biochemistry 1985, 24 (19), 5176-5181.

(39) Williams, R. S. B.; Cheng, L.; Mudge, A. W.; Harwood, A. J. A common mechanism of action for three mood-stabilizing drugs. Nature 2002, 417, 292. 